\title{
Tetramethylpyrazine Nitrone Improved Motor Deficits and Alleviated Dystrophic Muscle Pathology in the $m d x$ Mouse Model of Duchenne Muscular Dystrophy
}

\author{
Fengjiao Wang, ${ }^{2 *}$, Jing Wen ${ }^{1,2}{ }^{*}$, Guiliang Zhang ${ }^{1}$, Zheng Liu ${ }^{3,4}$, Haijing Zhong ${ }^{1}$, Gaoxiao Zhang1, \\ Yewei Sun1, Pei Yu1, Yuqiang Wang1, Zaijun Zhang1,2\# \\ ${ }^{1}$ Institute of New Drug Research, Jinan University College of Pharmacy, Guangzhou, China \\ ${ }^{2}$ International Cooperative Laboratory of Traditional Chinese Medicine Modernization and Innovative Drug Development of \\ Chinese Ministry of Education, Jinan University College of Pharmacy, Guangzhou, China \\ ${ }^{3}$ School of Stomatology and Medicine \& Foshan Stomatology Hospital, Foshan University, Foshan, China \\ ${ }^{4}$ Foshan Magpie Pharmaceuticals Co., LTD., Foshan, China \\ Email: "zaijunzhang@163.com
}

How to cite this paper: Wang, F.J., Wen, J., Zhang, G.L., Liu, Z., Zhong, H.J., Zhang, G.X., Sun, Y.W., Yu, P., Wang, Y.Q. and Zhang, Z.J. (2020) Tetramethylpyrazine Nitrone Improved Motor Deficits and Alleviated Dystrophic Muscle Pathology in the $m d x$ Mouse Model of Duchenne Muscular Dystrophy. Journal of Biosciences and Medicines, 8, 56-66.

https://doi.org/10.4236/jbm.2020.88006

Received: July 3, 2020

Accepted: August 10, 2020

Published: August 13, 2020

Copyright $\odot 2020$ by author(s) and Scientific Research Publishing Inc. This work is licensed under the Creative Commons Attribution International License (CC BY 4.0).

http://creativecommons.org/licenses/by/4.0/ (c) (i) Open Access

\begin{abstract}
Duchenne muscular dystrophy (DMD) is a lethal X-linked recessive neuromuscular disorder caused by mutations in the dystrophin encoding gene, with the characteristics of a severe and progressive destruction of muscle structure and function. Skeletal muscle fibrosis is one of the pathological features of DMD. Tetramethylpyrazine (2,3,5,6-tetramethylpyrazine, TMP) has been demonstrated to reduce heart and liver fibrosis. Meanwhile, previous studies showed that Tetramethylpyrazine nitrone (TBN), a nitrone derivative of TMP, has promising therapeutic effects in several neurodegenerative models and is more potent than TMP. In this study, we investigated the potential effect of TBN on the $m d x$ mouse model of DMD. Eight-week-old $m d x$ mice were administered with TBN $(30 \mathrm{mg} / \mathrm{kg}$ ) intragastrically twice daily, with deflazacort $(1 \mathrm{mg} / \mathrm{kg})$ once a day as a positive control, for a total of 24 weeks. Behavioral tests including pole-climbing open-field test were monitored every 4 weeks. Histopathological assessment was conducted in the gastrocnemius and diaphragm muscles. The effects of TBN on protein levels of dysferlin were measured by immunohistochemistry. TBN significantly reduced the climbing time in pole test and increased the total distance moved in an open-field test of $m d x$ mice. TBN attenuated fibrosis in the gastrocnemius and diaphragmatic muscles. In addition, TBN protected gastrocnemius muscle fibers via increasing expression of the dysferlin in $m d x$ mice. In conclusion,
\end{abstract}


this study demonstrated that TBN could improve the motor deficits and muscle pathology of $m d x$ mouse, and it is worth further exploring the mechanism of action of TBN for DMD treatment.

\section{Keywords}

Duchenne Muscular Dystrophy, Fibrosis, Dysferlin, TBN, $m d x$ Mouse

\section{Introduction}

Duchenne muscular dystrophy (DMD) is a lethal and incurable disease, which is the most common and severe form of muscular dystrophy in newborn. It results from X-linked defects in dystrophin gene, leading to life-threatening complications as respiratory and heart muscles are affected [1]. The latest data compiled from a global newborn screening study indicate that the prevalence rate is approximately 1:5000 for male newborns [2]. DMD patients usually present with muscle weakness at between 2 and 3 years of age, lose their ability to walk during childhood, and eventually succumb to respiratory and cardiac failure in their teens or early twenties [3]. Clinically studied treatment methods include glucocorticoid therapy, gene therapy, and stem-cell therapy. The only drug that can effectively slow down the progression of the disease is deflazacort, a corticosteroid, which was approved by the US FDA in 2016. However, long-term usage of deflazacort would cause a series of adverse reactions such as weight gain, immunosuppression, behavioral disorders, and cataracts [4]. New drugs or therapies with fewer adverse effects for DMD treatment are still urgently needed.

The $m d x$ mouse bearing a closer resemblance to human DMD pathology was recommended as the model for preclinical tests and proof-of-concept studies [5]. In the $m d x$ mouse model, muscle degeneration, necrosis and fibrosis are caused by dysfunction of the sarcolemma [6]. Fibrosis contributes directly to muscular dysfunction and inhibits muscle regeneration in $m d x$ mouse. And, skeletal muscle fibrosis is a characteristic of muscle dystrophies [7]. Disrupting calcium homeostasis, primarily calcium overload, and increased oxidative stress are hallmarks of dystrophic muscle [8].

TMP, the major active ingredient of Ligusticum wallichii Franchat (Chuanxiong), demonstrated activity to reduce liver fibrosis in a previous study [9]. TMP attenuated gastrocnemius (GAS) muscle atrophy by increasing the suppression of $\mathrm{Ca}^{2+} /$ reactive oxygen species [10]. TBN (2-[[(1,1-dimethylethyl)oxidoimino]-methyl]-3,5,6-trimethylpyrazine) is a novel nitrone derivative of TMP with remarkable effects on calcium overload blockade, free radical-scavenging and mitochondrial oxidative stress reduction [11] [12]. Additionally, TBN could maintain mitochondrial function, improve motor system function and skeletal muscle coordination in vivo [13]. Taking advantage of the beneficial effects of TBN, therefore, we investigated the potential effects of TBN on the $m d x$ mouse model of Duchenne muscular dystrophy. 


\section{Materials and Methods}

\subsection{Reagents and Antibodies}

Deflazacort was purchased from Beijing Puyihua Technology Co., Ltd. (Beijing, China). 0.01 M PBS (pH 7.2) was purchased from Wuhan Servicebio Technology Co., Ltd. (Wuhan, China). The hematoxylin-eosin (H \& E) staining kit was purchased from Nanjing Institute of Biological Engineering (Nanjing, China). Masson tricolor dyeing kit was purchased from Solarbio (Beijing, China). Anti-dysferlin antibody (ab124684) was purchased from Abcam (Cambridge, MA, USA).

\subsection{Animals}

Seven-week-old male mice, including both wild-type controls (C57BL/10ScSn) and the $m d x$ mice on a C57BL/10ScSn background, were purchased from Nanjing Biomedical Research Institute (NBRI) of Nanjing University, China. All mice were housed 4 - 6 per cage at room temperature with a 12-hour night-day cycle and were fed with pellets and water ad libitum. Mice were allowed to adapt to the environment for 1 week before beginning experimentation. All animal procedures were performed in accordance with the US NIH's "Guide for the Care and Use of Laboratory Animals" and approved by the Animal Ethics Committee of the Guangzhou University of Chinese Medicine, China (approved number \# 20181104002).

\subsection{Experimental Design}

For a 24-week course of therapy, male mice ( $\mathrm{n}=43$; 8 weeks of age) were randomly divided into 4 groups: the wild type group $(n=11$, saline, twice a day, gavage), the model group ( $\mathrm{n}=12$, saline, twice a day, gavage), the TBN treatment group $(\mathrm{n}=10,30 \mathrm{mg} / \mathrm{kg}$, twice a day, gavage as previously reported [11]) and the deflazacort treatment group (DFZ, $\mathrm{n}=10,1 \mathrm{mg} / \mathrm{kg}$, once a day, ip given as previously reported [14]). The reason we chose TBN $(30 \mathrm{mg} / \mathrm{kg})$ was that in the previously study for the therapeutic effects of TBN $(10 \mathrm{mg} / \mathrm{kg}, 30 \mathrm{mg} / \mathrm{kg}, 60$ $\mathrm{mg} / \mathrm{kg}$ ) on SOD1 ${ }^{\mathrm{G} 93 \mathrm{~A}}$ mouse model of amyotrophic lateral sclerosis, TBN (30 $\mathrm{mg} / \mathrm{kg}$ ) had the most obvious improvement in the behavior and fibrosis of SOD $1^{\text {G93A }}$ mice (Not yet published). At the end of study, diaphragm (DIA) and gastrocnemius (GAS) muscles were collected for pathological evaluation because DMD present with progressive muscle degeneration of respiratory (DIA) and appendicular (GAS) muscles [15].

\subsection{Behavioral Tests}

To evaluate the effects of TBN on motor coordination and spontaneous locomotor activity, the pole-climbing and open-field tests were performed. Behavioral tests were performed every 4 weeks after 8 weeks of drug administration. Open-field test was used to assess the locomotor and exploratory activity of animals in a new environment. Motor function was also evaluated with a pole-climbing test. 
Open-field test and pole-climbing test were conducted according to our previous publication [16].

\subsection{Isolation and Preparation of Gastrocnemius and Diaphragm}

Thirty-two-week-old $m d x$ mice and WT mice were euthanized with $0.1 \%$ pentobarbital sodium $(0.15 \mathrm{~mL} / 10 \mathrm{~g})$. After perfusion with ice cold $0.01 \mathrm{M} \mathrm{PBS}$ ( $\mathrm{pH}$ 7.2 ), the muscles were isolated. The left part of each muscle specimen was removed and stored at $-80^{\circ} \mathrm{C}$ while the right part was fixed with paraformaldehyde for 24 hours and embedded in paraffin.

\subsection{Immunohistochemistry Analysis for Dysferlin}

To reveal sarcolemmal dysferlin expression, the transverse section of the gastrocnemius was treated with $3 \% \mathrm{H}_{2} \mathrm{O}_{2}$ for $10 \mathrm{~min}$. to quench endogenous peroxidase activity. It was then blocked for $1.5 \mathrm{~h}$ in $1 \%$ Triton, $10 \%$ normal bovine serum (FBS) in PBS at room temperature. The slides were then incubated with rabbit-anti-dysferlin (1:200 dilution) at $4^{\circ} \mathrm{C}$ overnight, followed by incubation with the appropriate conjugated secondary antibodies for $1 \mathrm{~h}$. Tissue coloration with 3,3-diaminobenzidine was observed under an inverted microscope (Zeiss, Germany), then finally blocked with a neutral resin. Quantitative analyses of the images in gray scale were performed using Image-Pro Plus 6.0. Mean integrated optical density was plotted on the abscissa, and groups were plotted on the ordinate for statistics.

\subsection{Histological Staining and Analysis}

Paraffin-embedded gastrocnemius and diaphragm tissues were cut into 4- $\mu \mathrm{m}$ sections and dried at $37^{\circ} \mathrm{C}$ overnight. $\mathrm{H} \& \mathrm{E}$ and Masson staining were conducted according to our previous report [17]. Histological images were acquired on a digital scanning microscopy imaging system (M8, Precipoint, Germany) under $20 \times$ and $40 \times$ objectives. The percentage of the blue area to entire tissue area, which reflects the level of fibrosis, was calculated by Materials Image Processing and Automated Reconstruction (MIPAR ${ }^{\mathrm{TM}}$ ) software [18].

\subsection{Statistics}

Data were presented as mean \pm SEM. One-way analysis of variance (ANOVA) or two-way ANOVA followed by Tukey's multiple comparison test was performed to compare all treatment groups using Prism (GraphPad6.0) software. Differences with $p<0.05$ were considered significant.

\section{Results}

\subsection{TBN Improves Behavioral Deficits in $m d x$ Mice}

The $m d x$ mice exhibited remarkably prolonged climbing times starting from eight weeks of administration, which indicated that the $m d x$ mice had decreased motor coordination ability compared with that of age-matched WT mice 
(Figure 1(A)). Deflazacort improved $m d x$ mice's uncoordinated movement during the 24 weeks of treatment. Administration of TBN from the eighth week until the $24^{\text {th }}$ also significantly $(p<0.05)$ alleviated the mice's deteriorating coordination. In the open-field test, $m d x$ mice displayed a significant reduction in total distance moved when compare to the WT mice group after 12 weeks of experimentation; the distance moved continued to decrease with time (Figure $1(B))$. Treatment with TBN significantly $(p<0.05)$ improved the total distance moved by the $m d x$ mice and increased their spontaneous movement.

\subsection{Effect of TBN on Muscle Fiber Size in $m d x$ Mice}

After H\&E staining (Figure 2(A)), the fiber cross-sectional area of the gastrocnemius muscle was measured. The percentage of muscle fibers with cross-sectional areas of $<200 \mu \mathrm{m}^{2}$ was higher in $m d x$ mice than in WT mice (Figure 2(B)). After TBN's administration, the percentage of muscle fibers less than $200 \mu \mathrm{m}^{2}$ decreased and the proportion of muscle fibers greater than $200 \mu \mathrm{m}^{2}$ in cross-sectional area increased, similar to the DFZ group.

\subsection{Effect of TBN on Dystrophic Skeletal Muscles of $m d x$ Mice}

The amount of fibrosis in the gastrocnemius and diaphragmatic muscles in $m d x$ mice was greater compared to that of the WT mice group (Figure $3(\mathrm{~A})$ and

A

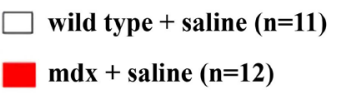

$\mathrm{mdx}+$ TBN $30 \mathrm{mg} / \mathrm{kg}(\mathrm{m}=10)$
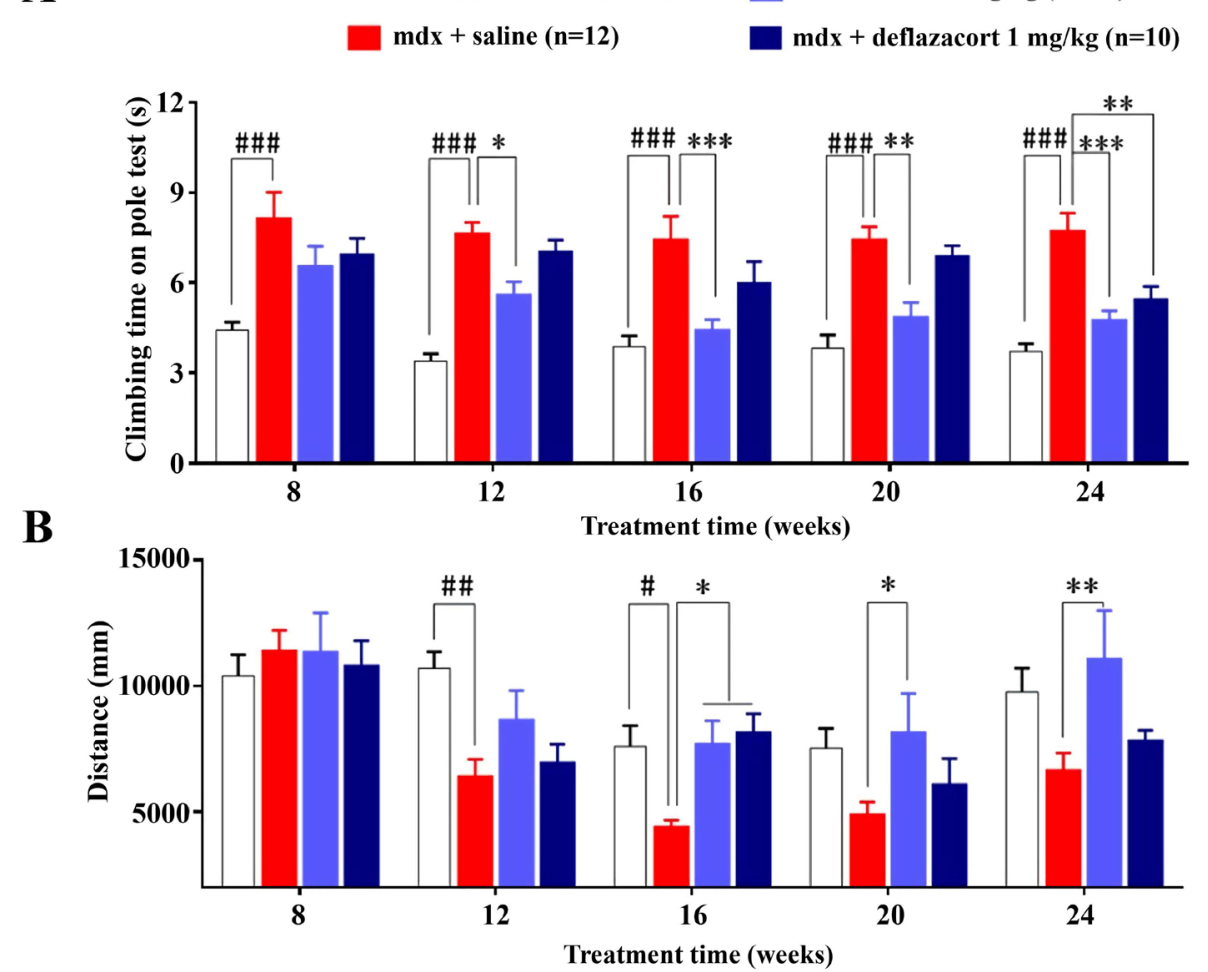

Figure 1. TBN improves behavioral deficits in $m d x$ mice. (A) Climbing time on pole test; (B) total distance traveled in open-field test. Data are expressed as mean \pm SEM, ${ }^{\#} P<0.05$, ${ }^{\# \#} P<0.01$ and ${ }^{\# \# \#} P<0.001$ relative to wild type mice; ${ }^{\star} P<0.05,{ }^{* *} P<0.01$ and ${ }^{* * *} P<0.001$ relative to $m d x$ mice, analyzed by two-way ANOVA with Tukey's multiple comparisons test. 
A

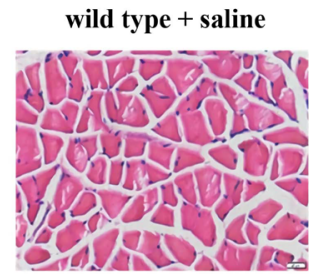

mdx + saline

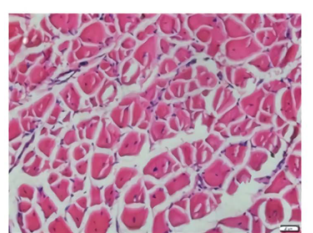

mdx + TBN

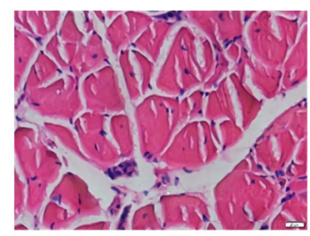

mdx + deflazacort

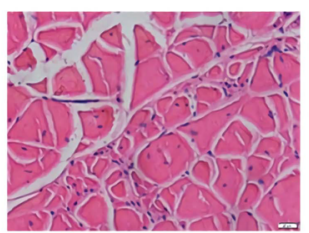

B
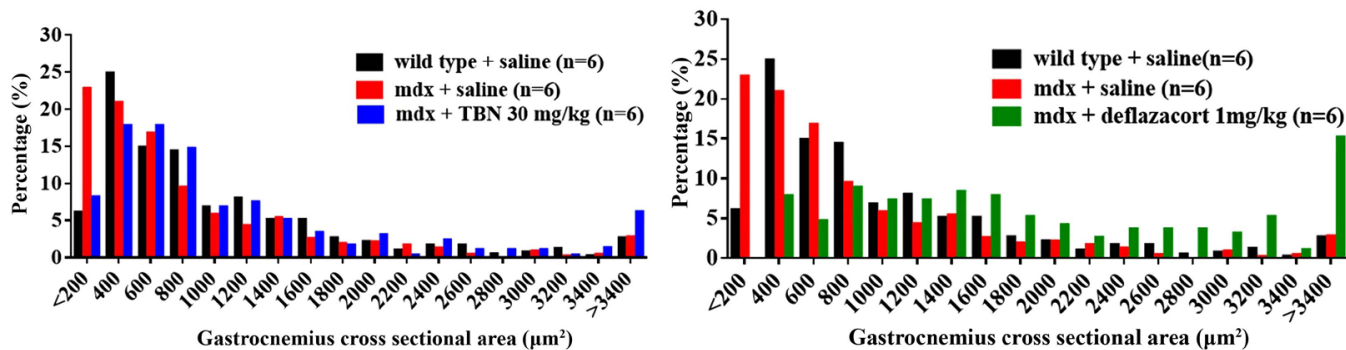

Figure 2. TBN attenuates the decrease in muscle fiber size of $m d x$ mice. (A) Representative figures of cross-sectional muscle fibers by H\&E staining. (B, C) Distribution of cross-sectional areas of gastrocnemius muscle fibers in wild-type, model, TBN-treated and DFZ-treated mice. Each group is comprised of six mice.

$\mathbf{A}$

wild type + saline

mdx + saline

mdx + TBN

mdx + deflazacort
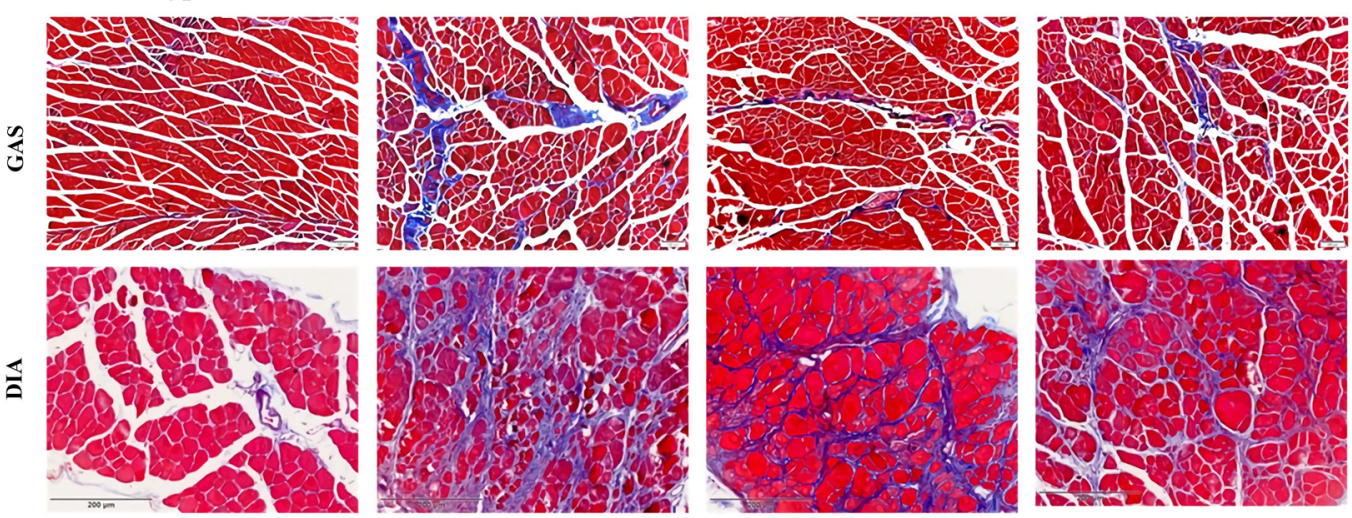

C

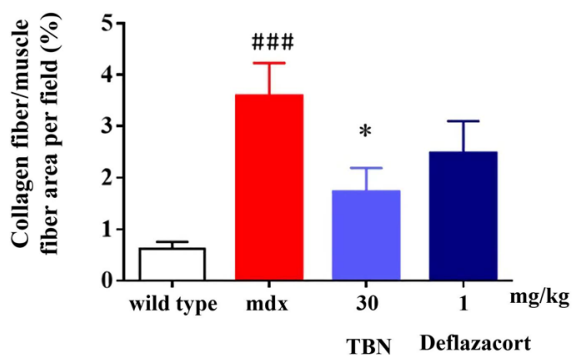

D

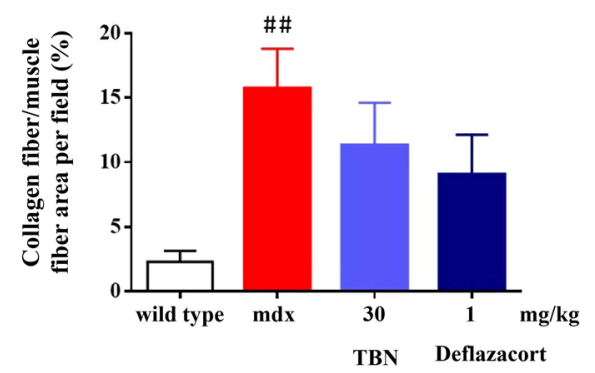

Figure 3. TBN reduces fibrosis in dystrophic skeletal muscle of $m d x$ mice. (A, B) The Masson staining images of gastrocnemius (GAS) and diaphragm (DIA) muscles in different mice groups. The extent of total collagen staining (blue) in GAS and DIA were used as a marker of fibrosis. Scale bar $=200 \mu \mathrm{m}$. (C, D) Quantitative analysis of the total collagen staining of the gastrocnemius (C) and diaphragm (D). Data are expressed as mean \pm SEM, ${ }^{\# \#} P<0.01$ and ${ }^{\# \#} P<0.001$ relative to wild type mice; ${ }^{\star} P<0.05$ relative to $m d x$ mice, analyzed by one-way ANOVA with Tukey's multiple comparisons test. Each group is comprised of six mice. 
Figure 3(B)). Treatment with TBN significantly $(p<0.05)$ attenuated fibrosis in the GAS (Figure $3(\mathrm{C})$ ). However, the effect of TBN on fibrosis in the DIA was no statistically $(p>0.05)$ difference (Figure $3(D))$.

\subsection{Effect of TBN on Dysferlin Expression on the Surface of the Gastrocnemius Muscle Cell Membrane}

Immunohistochemical staining was performed to assess dysferlin abundance in the gastrocnemius (Figure 4(A)). Histological comparison of the $m d x$ mice and WT mice, indicated that the amount of dysferlin on the surface of the gastrocnemius muscle cell membrane was reduced in the $m d x$ group. The expression of dysferlin in TBN-treated mice was higher than that in saline-treated $m d x$ mice.

\section{Discussion}

The open-field test and pole test are experimental test used to evaluate general locomotor activity levels [19]. Muscle atrophy and necrosis were evaluated by analyzing the cross-sectional area of the gastrocnemius muscle in mice [20]. Simultaneously, ameliorating muscle fibrosis has been considered a viable approach for DMD treatment [21]. Thus, Masson staining was used to assess the fibrosis status of gastrocnemius and diaphragm [22]. In addition, dysferlin plays an important role in membrane fusion and muscle membrane repair in skeletal muscle fibers [23].

TBN treatment improved motor function in $m d x$ mice and enhanced both spontaneous motion and motor coordination in $m d x$ mice. Then, TBN improved muscle morphological change in $m d x$ mice and reduced the proportion of muscle fibers with a cross-sectional area less than $200 \mu \mathrm{m}^{2}$, suggesting that TBN can

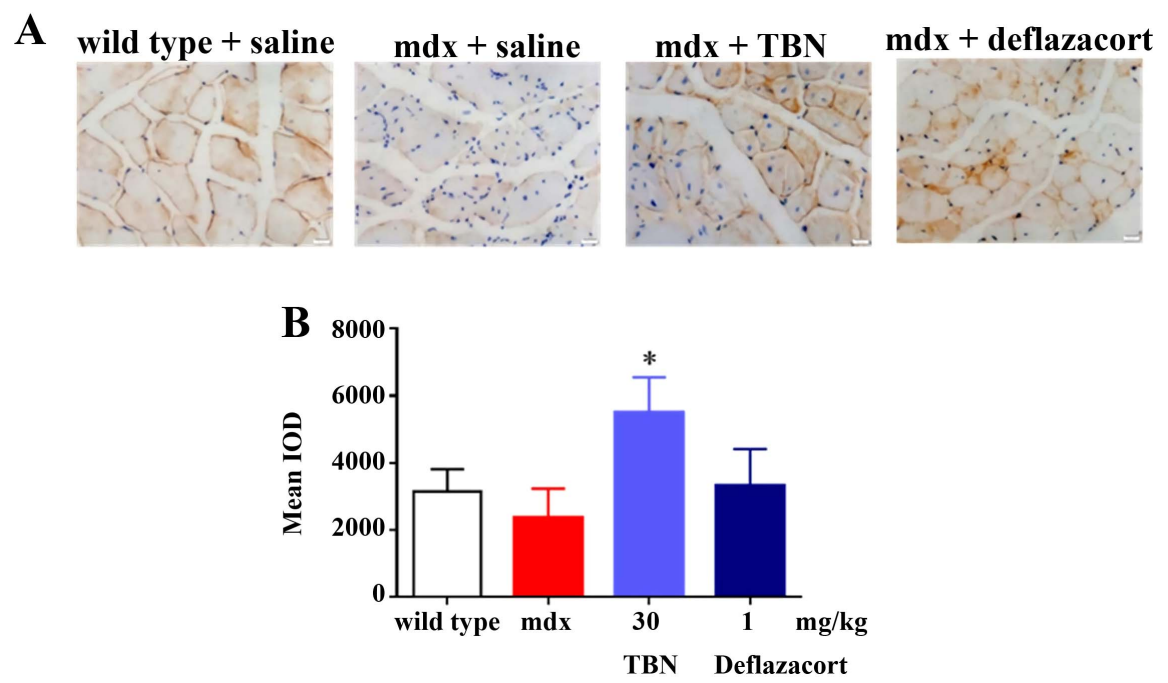

Figure 4. TBN increases dysferlin expression in gastrocnemius of $m d x$ mice. (A) Representative images of dysferlin staining in gastrocnemius by immunohistochemistry. (B) Quantitative analysis of dysferlin expression on sarcolemmal surface. Data are expressed as mean $\pm \mathrm{SEM},{ }^{\star} P<0.05$ relative to $m d x$ mice, analyzed by one-way ANOVA with Tukey's multiple comparisons test. Each group is comprised of six mice. 
reduce the atrophy of gastrocnemius muscle fibers. In addition, TBN significantly attenuated fibrosis in the gastrocnemius and increased dysferlin expression on the surface of the gastrocnemius muscle cell membrane.

The mdx mouse model is widely used for DMD research as it presents similar skeletal muscle pathology to DMD patients at 3 to 8 weeks of age [24]. In addition to being valuable for screening anti-nutritional drugs, mdx mice lack functional dystrophin, much like human DMD. The experiment was designed to select eight-week-old mice for treatment for 24 weeks to better evaluate the efficacy. Due to the complexity of the molecular pathways involved in the pathophysiology of disease progression, it is difficult to identify all molecular participants involved in DMD pathology. Alter et al. [25] have shown that morpholino phosphorodiamidate AONs induce expression of functional dystrophin in body-wide skeletal muscles of the dystrophic mdx mouse, resulting in improved muscle function. Nelson et al. [26] have shown that delivering the CRISPR-Cas9 system to the $m d x$ mouse model of DMD with adeno-associated virus can remove the mutated exon 23 from the dystrophin gene and increase dystrophin expression, thus also improving muscle function. Improving muscular fibrosis may also be a viable treatment to improve overall muscle condition [21].

TBN, a derivative of TMP, has a powerful free radical scavenging nitrone moiety that can not only improve skeletal muscle fibrosis, regulate inflammation, protect nerves, and improve animal behavioral disorders, but also reverse the expression of apoptosis-related proteins [11] [12] [13]. In this study, our data indicates that TBN treatment provides significant behavioral improvements in $m d x$ mouse over the existing DMD treatment. We speculate that TBN may protect muscle functions by ameliorating muscle fibrosis and facilitating muscle membrane repair through dysferlin improvement. Previous studies identified that TBN is a powerful free radical scavenger against oxidative stress [12] [13] Moreover, TBN could maintain calcium homeostasis and mitochondrial function, improve motor system function and skeletal muscle coordination in vivo [12] [27] [28] The improvement in muscular function in the mdx mouse model of DMD exhibited by TBN could be attributed, at least partly, to the calcium overload blockage and mitochondrial function improvement. However, more mechanistic studies of TBN need to be conducted.

\section{Conclusion}

In conclusion, TBN improved motor function, reduced muscle fibrosis, and improved the atrophy of the gastrocnemius muscle in the $m d x$ mouse. These findings suggest that TBN may be effective in the treatment of muscular dystrophy.

\section{Source of Funding}

This work was partly supported by grants from the Natural Science Fund of China (81872842, 31861163001 and U1801287), the Foshan Innovative Science Project

(2017IT100153) and the China Innovative Drug Project (2019ZX09301172). 


\section{Conflicts of Interest}

The authors declare no conflicts of interest.

\section{References}

[1] Finsterer, J. (2006) Cardiopulmonary Support in Duchenne Muscular Dystrophy. Lung, 184, 205-215. https://doi.org/10.1007/s00408-005-2584-X

[2] Mendell, J.R., Shilling, C., Leslie, N.D., Flanigan, K.M., al-Dahhak, R., Gastier-Foster, J., Kneile, K., Dunn, D.M., Duval, B., Aoyagi, A., Hamil, C., Mahmoud, M., Roush, K., Bird, L., Rankin, C., Lilly, H., Street, N., Chandrasekar, R. and Weiss, R.B. (2012) Evidence-Based Path to Newborn Screening for Duchenne Muscular Dystrophy. Annals of Neurology, 71, 304-313. https://doi.org/10.1002/ana.23528

[3] Mendell, J.R. and Lloyd-Puryear, M. (2013) Report of MDA Muscle Disease Symposium on Newborn Screening for Duchenne Muscular Dystrophy. Muscle Nerve, 48 , 21-26. https://doi.org/10.1002/mus.23810

[4] Beytía, M.A., Vry, J. and Kirschner, J. (2012) Drug Treatment of Duchenne Muscular Dystrophy: Available Evidence and Perspectives. Acta Myologica, 31, 4-8.

[5] Bulfield, G., Siller, W.G., Wight, P.A. and Moore, K.J. (1984) X Chromosome-Linked Muscular Dystrophy ( $\mathrm{mdx}$ ) in the Mouse. Proceedings of the National Academy of Sciences, 81, 1189-1192. https://doi.org/10.1073/pnas.81.4.1189

[6] Han, R., Rader, E.P., Levy, J.R., Bansal, D. and Campbell, K.P. (2011) Dystrophin Deficiency Exacerbates Skeletal Muscle Pathology in Dysferlin-Null Mice. Skeletal Muscle, 1, 35. https://doi.org/10.1186/2044-5040-1-35

[7] Klingler, W., Jurkat-Rott, K., Lehmann-Horn, F. and Schleip, R. (2012) The Role of Fibrosis in Duchenne Muscular Dystrophy. Acta Myologica, 31, 184-195.

[8] Pal, R., Palmieri, M., Loehr, J.A., Li, S., Abo-Zahrah, R., Monroe, T.O., Thakur, P.B., Sardiello, M. and Rodney, G.G. (2014) Src-Dependent Impairment of Autophagy by Oxidative Stress in a Mouse Model of Duchenne Muscular Dystrophy. Nature Communications, 5, 4425. https://doi.org/10.1038/ncomms5425

[9] Huang, C., Wu, X., Wang, S., Wang, W., Guo, F., Chen, Y., Pan, B., Zhang, M. and Fan, X. (2018) Combination of Salvia miltiorrhiza and Ligustrazine Attenuates Bleomycin-Induced Pulmonary Fibrosis in Rats via Modulating TNF-Alpha and TGF-Beta. Chinese Medicine, 13, 36. https://doi.org/10.1186/s13020-018-0194-9

[10] Hu, N.-F., Chang, H., Du, B., Zhang, Q.-W., Arfat, Y., Dang, K. and Gao, Y.-F. (2016) Tetramethylpyrazine Ameliorated Disuse-Induced Gastrocnemius Muscle Atrophy in Hindlimb Unloading Rats through Suppression of Ca2+/ROS-Mediated Apoptosis. Applied Physiology, Nutrition, and Metabolism, 42, 117-127. https://doi.org/10.1139/apnm-2016-0363

[11] Guo, B., Xu, D., Duan, H., Du, J., Zhang, Z., Lee, S.M. and Wang, Y. (2014) Therapeutic Effects of Multifunctional Tetramethylpyrazine Nitrone on Models of Parkinson's Disease in Vitro and in Vivo. Biological and Pharmaceutical Bulletin, 37, 274-285. https://doi.org/10.1248/bpb.b13-00743

[12] Sun, Y., Yu, P., Zhang, G., Wang, L., Zhong, H., Zhai, Z., Wang, L. and Wang, Y. (2012) Therapeutic Effects of Tetramethylpyrazine Nitrone in Rat Ischemic Stroke Models. Journal of Neuroscience Research, 90, 1662-1669. https://doi.org/10.1002/jnr.23034

[13] Zhang, Z., Zhang, G., Sun, Y., Szeto, S.S.W., Law, H.C.H., Quan, Q., Li, G., Yu, P., Sho, E., Siu, M.K.W., Lee, S.M.Y., Chu, I.K. and Wang, Y. (2016) Tetramethylpyrazine Nitrone, a Multifunctional Neuroprotective Agent for Ischemic Stroke Thera- 
py. Scientific Reports, 6, Article No. 37148. https://doi.org/10.1038/srep37148

[14] Quattrocelli, M., Barefield, D.Y., Warner, J.L., Vo, A.H., Hadhazy, M., Earley, J.U., Demonbreun, A.R. and McNally, E.M. (2017) Intermittent Glucocorticoid Steroid Dosing Enhances Muscle Repair without Eliciting Muscle Atrophy. Journal of Clinical Investigation, 127, 2418-2432. https://doi.org/10.1172/JCI91445

[15] Apolinário, L.M., De Carvalho, S.C., Santo Neto, H. and Marques, M.J. (2015) Long-Term Therapy with Omega-3 Ameliorates Myonecrosis and Benefits Skeletal Muscle Regeneration in Mdx Mice. The Anatomical Record, 298, 1589-1596. https://doi.org/10.1002/ar.23177

[16] Xu, B., Zheng, C., Chen, X., Zhang, Z., Liu, J., Spencer, P. and Yang, X. (2019) Dysregulation of Myosin Complex and Striated Muscle Contraction Pathway in the Brains of ALS-SOD1 Model Mice. ACS Chemical Neuroscience, 10, 2408-2417. https://doi.org/10.1021/acschemneuro.8b00704

[17] Yang, Y., Yan, H., Jing, M., Zhang, Z., Zhang, G., Sun, Y., Shan, L., Yu, P., Wang, Y. and $\mathrm{Xu}, \mathrm{L}$. (2016) Andrographolide Derivative AL-1 Ameliorates TNBS-Induced Colitis in Mice: Involvement of NF- $\kappa \mathrm{B}$ and PPAR- $\gamma$ Signaling Pathways. Scientific Reports, 6, Article No. 29716. https://doi.org/10.1038/srep29716

[18] Sosa, J.M., Huber, D.E., Welk, B. and Fraser, H.L. (2014) Development and Application of MIPAR ${ }^{\mathrm{m}}$ : A Novel Software Package for Two- and Three-Dimensional Microstructural Characterization. Integrating Materials and Manufacturing Innovation, 3, 123-140. https://doi.org/10.1186/2193-9772-3-10

[19] Wu, L., Su, Z., Zha, L., Zhu, Z., Liu, W., Sun, Y., Yu, P., Wang, Y., Zhang, G. and Zhang, Z. (2019) Tetramethylpyrazine Nitrone Reduces Oxidative Stress to Alleviate Cerebral Vasospasm in Experimental Subarachnoid Hemorrhage Models. NeuroMolecular Medicine, 21, 262-274. https://doi.org/10.1007/s12017-019-08543-9

[20] Karpati, G., Carpenter, S. and Prescott, S. (1988) Small-Caliber Skeletal Muscle Fibers Do Not Suffer Necrosis in Mdx Mouse Dystrophy. Muscle \& Nerve, 11, 795-803. https://doi.org/10.1002/mus.880110802

[21] Zhou, L. and Lu, H. (2010) Targeting Fibrosis in Duchenne Muscular Dystrophy. Journal of Neuropathology \& Experimental Neurology, 69, 771-776. https://doi.org/10.1097/NEN.0b013e3181e9a34b

[22] Wagner, K.R., McPherron, A.C., Winik, N. and Lee, S.J. (2002) Loss of Myostatin Attenuates Severity of Muscular Dystrophy in Mdx Mice. Annals of Neurology: Official Journal of the American Neurological Association and the Child Neurology Society, 52, 832-836. https://doi.org/10.1002/ana.10385

[23] Bansal, D. and Campbell, K.P. (2004) Dysferlin and the Plasma Membrane Repair in Muscular Dystrophy. Trends in Cell Biology, 14, 206-213.

https://doi.org/10.1016/j.tcb.2004.03.001

[24] Sun, C.-C., Li, S.-J., Yang, C.-L., Xue, R.-L., Xi, Y.-Y., Wang, L., Zhao, Q.-L. and Li, D.-J. (2015) Sulforaphane Attenuates Muscle Inflammation in Dystrophin-Deficient Mdx Mice via NF-E2-Related Factor 2 (Nrf2)-Mediated Inhibition of NF- $\kappa$ B Signaling Pathway. Journal of Biological Chemistry, 290, 17784-17795. https://doi.org/10.1074/jbc.M115.655019

[25] Alter, J., Lou, F., Rabinowitz, A., Yin, H., Rosenfeld, J., Wilton, S.D., Partridge, T.A. and Lu, Q.L. (2006) Systemic Delivery of Morpholino Oligonucleotide Restores Dystrophin Expression Bodywide and Improves Dystrophic Pathology. Nature Medicine, 12, 175-177. https://doi.org/10.1038/nm1345

[26] Nelson, C.E., Hakim, C.H., Ousterout, D.G., Thakore, P.I., Moreb, E.A., Rivera, 
R.M.C., Madhavan, S., Pan, X., Ran, F.A., Yan, W.X., Asokan, A., Zhang, F., Duan, D. and Gersbach, C.A. (2016) In Vivo Genome Editing Improves Muscle Function in a Mouse Model of Duchenne Muscular Dystrophy. Science, 351, 403-407. https://doi.org/10.1126/science.aad5143

[27] Zhu, L., Sun, Y., Zhang, G., Yu, P., Wang, Y. and Zhang, Z. (2015) Radical-Scavenging and Anti-Oxidative Activities of TBN in Cell-Free System and $\mathrm{Mu}$ rine H9c2 Cardiomyoblast Cells. Journal of Antioxidant Activity, 1, 55. https://doi.org/10.14302/issn.2471-2140.jaa-15-765

[28] Zhang, T., Gu, J., Wu, L., Li, N., Sun, Y., Yu, P., Wang, Y., Zhang, G. and Zhang, Z. (2017) Neuroprotective and Axonal Outgrowth-Promoting Effects of Tetramethylpyrazine Nitrone in Chronic Cerebral Hypoperfusion Rats and Primary Hippocampal Neurons Exposed to Hypoxia. Neuropharmacology, 118, 137-147.

https://doi.org/10.1016/j.neuropharm.2017.03.022 